\title{
Síndrome de la uña verde: revisión actualizada de una patología emergente en la era COVID-19
}

\section{Green nail syndrome: updated review of an emerging disease in the era of COVID-19}

\author{
Dra. Catalina Hasbún Z. ${ }^{1 *}$ \\ Dr. Rodrigo Cruz C. ${ }^{2}$ \\ ${ }^{1}$ Residente de Dermatología, Universidad de los Andes, Santiago, Chile. \\ ${ }^{2}$ Centro de Diagnóstico e Investigación de Enfermedades Infecciosas (CDIEI), Universidad de \\ Valparaíso, Valparaíso, Chile. \\ *Autor para correspondencia: cphasbun@miuandes.cl \\ RECIBIDO: 10 de septiembre 2021 \\ APROBADO: 20 de octubre 2021
}

DOI: $10.22370 /$ bolmicol.2021.36.2.3043

Palabras clave: cloroniquia, Pseudomonas aeruginosa, síndrome de uña verde.

Keywords: chloronychia, Pseudomonas aeruginosa, green nail syndrome.

\section{RESUMEN}

El síndrome de la uña verde o cloroniquia corresponde a la infección por Pseudomonas aeruginosa de una lámina ungueal dañada en pacientes con algún factor de riesgo identificable, siendo los más frecuentes la inmunosupresión, el ambiente húmedo constante y la patología ungueal preexistente. $\mathrm{Su}$ diagnóstico es relativamente sencillo si se logra observar la tríada característica de coloración verdosa de la lámina ungueal, paroniquia proximal crónica y onicolisis distal; en casos de duda diagnóstica se puede enviar una muestra de la uña afectada para cultivos o estudio histopatológico. El pilar de su tratamiento corresponde al uso de antibióticos tópicos o sistémicos en conjunto con medidas generales que protejan de la humedad. Es muy importante enfatizar la prevención de esta patología en el personal de salud, especialmente en el contexto del lavado de manos frecuente y riguroso implementado durante la pandemia COVID-19, ya que existen reportes de transmisión nosocomial de P.aeruginosa por profesionales de la salud con infección ungueal.

\section{ABSTRACT}

Green nail syndrome or chloronychia is the infection of a damaged nail plate by Pseudomonas aeruginosa in a patient with an identifiable risk factor; the most frequently described are immunosuppression, a persistent 
Síndrome de la uña verde - Hasbún C. et al.

moist environment and preexisting nail disease. Its diagnosis is relatively simple if the characteristic triad of green discoloration of the nail plate, chronic proximal paronychia and distal onycholysis can be observed, in cases of doubt a sample of the affected nail can be sent for cultures or histopathology. The cornerstone of treatment is the use of topical or systemic antibiotics along with measures to protect the nail from moisture. Prevention of this disease must be emphasized in health care personnel, especially in the context of frequent and rigorous handwashing practices implemented during the COVID-19 pandemic, since there are reports of nosocomial transmission of P.aeruginosa by health care professionals with nail infection.

\section{INTRODUCCIÓN}

Pseudomonas aeruginosa es un patógeno oportunista que suele aislarse en medios naturales húmedos (agua, suelo, plantas) y en el medio intrahospitalario. ${ }^{1}$ Dentro de los hallazgos clínicos de la infección por este agente, las manifestaciones cutáneas son frecuentes, siendo secundarias a la inoculación local o a diseminación a la piel luego de una bacteriemia. El espectro de presentación es desde infecciones leves en pacientes inmunocompetentes hasta infecciones graves potencialmente mortales en personas inmunodeprimidas. ${ }^{2}$ Las infecciones cutáneas más frecuentes ocurren en pacientes sanos por la penetración de la bacteria a través de soluciones de continuidad, y corresponden al síndrome de la uña verde (SUV), intertrigo interdigital del pie, foliculitis, síndrome de pie caliente y otitis externa o del nadador. ${ }^{1,3}$

El SUV, cloroniquia o síndrome de FoxGoldman es secundario a la infección por $P$. aeruginosa de una lámina ungueal dañada y, en consecuencia, ocurre más frecuentemente en pacientes cuyas manos están constantemente expuestas al agua o que sufren de trauma mecánico repetitivo $\mathrm{u}$ otra causa de daño ungueal. ${ }^{4,5}$
Fue descrito inicialmente por los médicos Goldman y Fox en 1944, quienes fueron los primeros en asociar la pigmentación verdosa persistente de la uña con la infección por $P$. aeruginosa en 2 pacientes del ala de dermatología del Hospital General de Cincinnati (de allí su denominación de "síndrome de FoxGoldman") ${ }^{6}$. Esta observación fue seguida de múltiples reportes que mediante estudios bacteriológicos confirmaron este agente. ${ }^{7,8,9}$

Es importante destacar la posibilidad de infecciones nosocomiales por $P$. aeruginosa transmitidas por personal de salud con enfermedad ungueal demostrable ${ }^{10,11,12}$, así como también la contaminación de heridas quirúrgicas por infección de la uña del mismo paciente. $^{13}$

El objetivo de este artículo es una revisión breve de la patogénesis, clínica, diagnóstico y tratamiento del SUV, en el contexto del lavado de manos frecuente por parte de la comunidad y del personal de la salud durante la pandemia COVID.

\section{Patogenesis.}

$P$. aeruginosa es un bacilo gramnegativo móvil, aerobio estricto, considerado ubicuo en la naturaleza, que no forma parte de la microbiota cutánea normal. La mayoría de las cepas de esta bacteria producen pigmentos como la pioverdina y piocianina, responsables de la coloración típica de las uñas infectadas, que puede ser desde verde-amarillenta, azul o café-verdosa hasta negro-verdosa. ${ }^{1,2,4,14}$

Se ha postulado que el SUV corresponde a una paroniquia o infección del tejido blando que rodea a la uña; la separación de la lámina ungueal de su lecho permitiría la entrada de la bacteria, produciéndose la infección y la pigmentación característica de la lámina. ${ }^{4}$

Aunque se ha descrito que Pseudomonas corresponden a género de bacterias Gram negativas más frecuentemente aisladas de la 
Síndrome de la uña verde - Hasbún C. et al.

microbiota subungueal, la infección de una uña intacta es inusual, lo que apoya la necesidad de que exista algún otro factor gatillante en la patogénesis. ${ }^{4,15,16}$ Entre los factores de riesgo para esta patología se encuentran la inmunosupresión, el ambiente húmedo por una exposición prolongada a agua, jabones y detergentes y la patología ungueal (paroniquia, onicotilomanía, traumatismo local y psoriasis ungueal). Es por esto que se ha descrito más frecuentemente como una infección ocupacional, tanto en la comunidad como en el medio intrahospitalario, y como una infección oportunista en pacientes con patologías ungueales preexistentes.
Se ha descrito que la presencia de hongos favorece la colonización y potencia el crecimiento de $P$. aeruginosa en la uña, por lo que una onicomicosis concomitante también debe considerarse como un factor predisponente. ${ }^{2,17,18,19}$.Estudios de microscopía electrónica han demostrado la presencia de aperturas tipo túnel en la queratina de uñas coinfectadas con $P$. aeruginosa y Candida $s p$.; se postula que los hongos formarían estas estructuras que luego favorecerían la proliferación de las colonias bacterianas. ${ }^{20}$

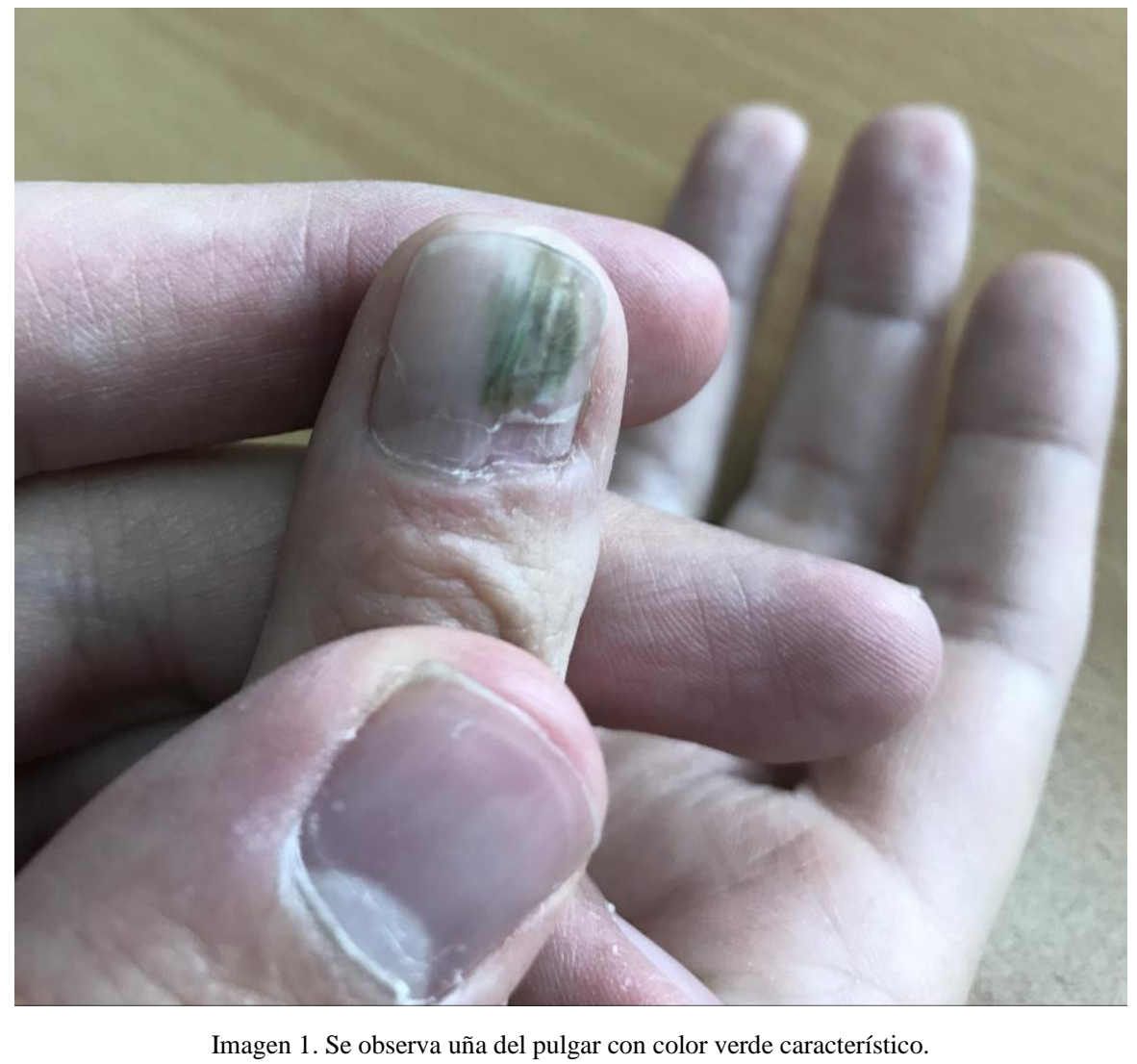

\section{Clínica.}

El SUV tiene una tríada clínica clásica que corresponde a la coloración verdosa de la lámina ungueal, paroniquia proximal crónica no sensible y onicolisis laterodistal (Imagen 1 y 2). ${ }^{2}$, $4,21,22$
Con respecto a esta forma de presentación, en una serie de 26 casos de Geizhals y Lipner se encontró onicolisis en un 76,9\% de los pacientes, coloración verdosa de la uña en un 38,5\% y coloración café-verdosa en un $30,8 \%{ }^{19}$ 
Síndrome de la uña verde - Hasbún C. et al.

El SUV suele observarse en una sola uña y aunque puede ocurrir en cualquiera de la mano o pie, el pulgar y el ortejo mayor son los más frecuentemente afectados. El compromiso de la lámina ungueal puede ser parcial o total $\mathrm{y}$ usualmente es indoloro. ${ }^{4,23,24,25}$
El diagnóstico diferencial a considerar en el SUV incluye el hematoma subungueal, melanoma maligno, infecciones por otros patógenos (Aspergillus, Candida, Proteus) y pigmentación exógena por sustancias químicas. ${ }^{4}$, 15

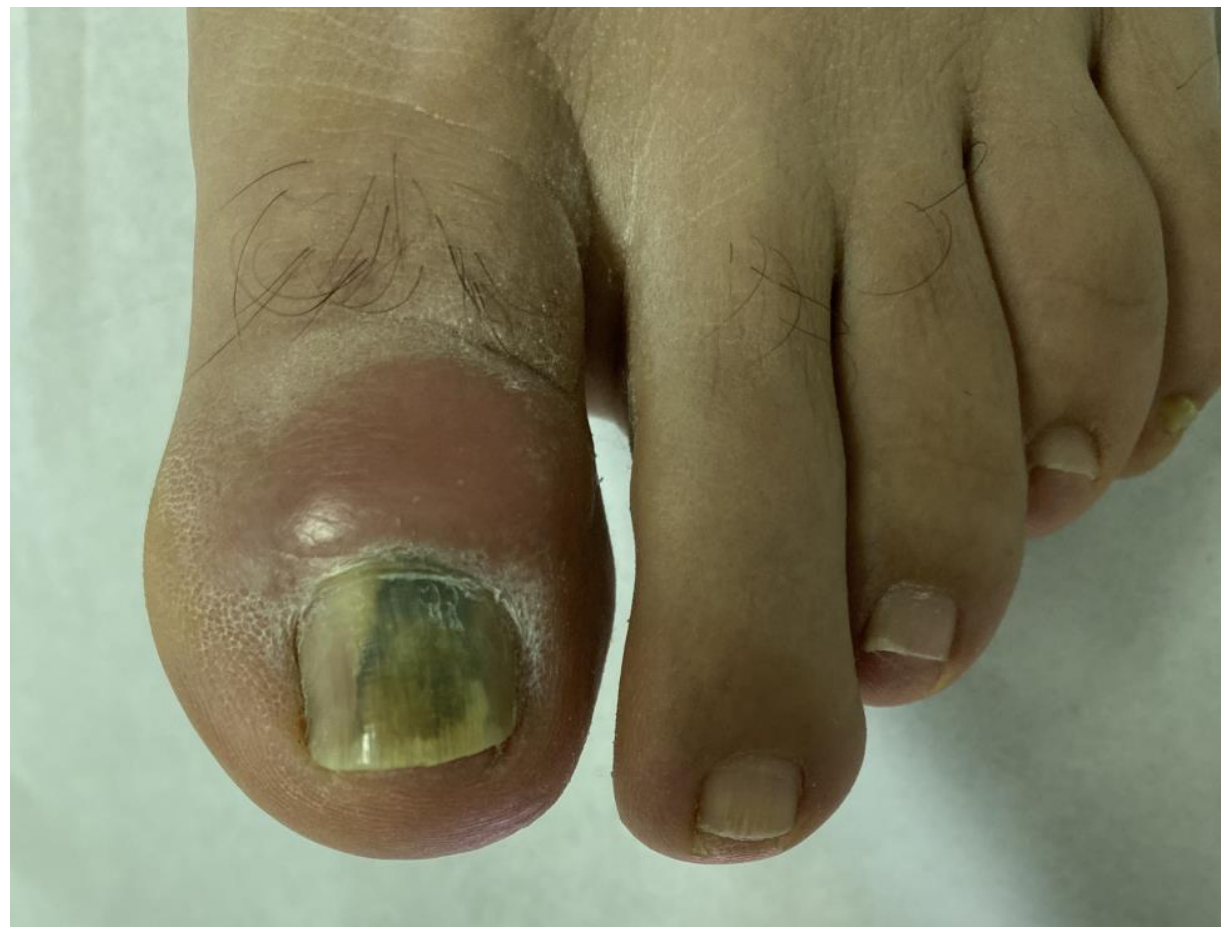

Imagen 2. Se observa uña del primer ortejo izquierdo con cloroniquia característica.

\section{Diagnóstico.}

La coloración verdosa de una lesión debe hacer sospechar la presencia de Pseudomonas Se ha sugerido la confirmación bacteriológica mediante el envío de muestras de la lámina o residuo subungueal de la uña afectada para cultivo y antibiograma, sin embargo, los resultados son inconsistentes en un alto porcentaje de los casos debido a la contaminación con bacterias comensales cutáneas, y la histopatología es muy poco sensible, por lo que la clínica suele ser suficiente para el diagnóstico de SUV. 1, 21, 23.

Es importante destacar que la coinfección fúngica puede estar subdiagnosticada en el SUV, ya que $P$. aeruginosa posee propiedades fungistáticas $y / 0$ fungicidas, habiéndose demostrado la inhibición del crecimiento in vitro tanto de levaduras como de hongos filamentosos por distintos mecanismos en presencia de la bacteria. ${ }^{17-19,26,27,28}$

\section{Tratamiento.}

No existen ensayos clínicos que permitan guiar las recomendaciones de tratamiento en SUV., ${ }^{41}$ La evidencia actual se basa en reportes y series de casos pequeñas.

En primer lugar, se pueden adoptar medidas generales como el corte o limpieza de la zona onicolítica y el uso de guantes para prevenir la inmersión repetida; la remoción quirúrgica de la lámina ungueal completa no se recomienda 
Síndrome de la uña verde - Hasbún C. et al.

actualmente, aunque puede ser necesaria en casos recalcitrantes. ${ }^{4,15,29,30}$

Se ha reportado exitosamente el uso de antisépticos locales como hipoclorito sódico, clorhexidina, ácido acético y dihidrocloruro de octenidina en compresas o baños de inmersión, sin embargo, debe considerarse que puede producirse irritación de la piel circundante. ${ }^{22,23,}$ 31,32

En cuanto al uso de antibióticos, se ha reportado eficacia con antibióticos tópicos incluyendo sulfadiazina de plata, aminoglucósidos (gentamicina y tobramicina en solución oftálmica), quinolonas (ciprofloxacino, ofloxacino, nadifloxacino), ungüento de neomicina/polimixina B y bacitracina. ${ }^{4}, 14,15,20$, 23, 30,32,33,34,35,36,37 Los antibióticos orales se recomiendan si hay falla a las terapias tópicas o en caso de preferencia del paciente, utilizándose un esquema de ciprofloxacino $500 \mathrm{mg}$ al día por 2 a 3 semanas, aunque en casos difíciles se recomienda la toma de cultivo y ajuste del tratamiento según antibiograma. ${ }^{4}, 15,23,38$ Es deseable la búsqueda de una onicomicosis concomitante, recordando que su tratamiento debe incluir antifúngicos orales en un curso prolongado. $2,4,38$

Para la prevención de infecciones nosocomiales transmitidas por el personal de salud se recomienda el examen periódico de las uñas, evitar el uso de esmaltes de colores que puedan ocultar la coloración verdosa típica del SUV y mantener las manos lo más secas posibles luego del lavado. ${ }^{38}$

\section{CONCLUSIÓN}

El síndrome de la uña verde o cloroniquia corresponde a la infección por $P$. aeruginosa de una uña previamente dañada en un paciente con algún factor de riesgo, dentro de los cuales la exposición constante al agua es uno de los más frecuentemente descritos, y que a su vez puede llevar a otras condiciones predisponentes para esta patología como onicomicosis y traumatismo ungueal crónico. La coloración típica de la uña permite aproximarse rápidamente al diagnóstico, de manera de implementar un tratamiento antibiótico adecuado. Los profesionales de la salud deben estar especialmente atentos, ya que corresponden a una de las poblaciones más afectadas por esta patología debido a la necesidad de un lavado de manos frecuente y riguroso, y también debido al riesgo de transmisión de $P$. aeruginosa a los pacientes hospitalizados, quienes son particularmente vulnerables al desarrollo de infecciones graves por este agente.

Conflictos de interés: Los autores declaran no tener conflictos de interés.

Agradecimientos: al Dr. Mathias Yagnam, del servicio de dermatología de la clínica Dávila por facilitar las fotografías de esta revisión.

\section{REFERENCIAS}

1. García-Martínez FJ, López-Martín I, Castellanos-González M, Segurado-Rodríguez MA. Green foot ulcers. Enferm Infecc Microbiol Clin. 2017; 35(8): 536-7.

2. Monteagudo B, Figueroa O, Suárez-Magdalena $\mathrm{O}$, Méndez-Lage S. Uña verde causada por onicomicosis coinfectada por Pseudomonas aeruginosa. Actas Dermosifiliogr. 2019; 110(9): 783-5.

3. Spernovasilis N, Psichogiou M, Poulakou G. Skin manifestations of Pseudomonas aeruginosa infections. Curr Opin Infect Dis. 2021; 34(2): 72-9.

4. Schwartz RA, Reynoso-Vasquez N, Kapila R. Chloronychia: The Goldman-Fox Syndrome Implications for Patients and Healthcare Workers. Indian J Dermatol. 2020; 65(1): 1-4. 
Síndrome de la uña verde - Hasbún C. et al.

5. Molina DN, Colón M, Bermúdez RH, RamírezRonda CH. Unusual presentations of Pseudomonas aeruginosa infections: a review. Bol Asoc Med P R. 1991; 83(4): 160-3.

6. Goldman L, Fox H. Greenish pigmentation of nail plates from Bacillus Pyocyaneus infection. AMA Arch Dermatol Syphilol. 1944; 68: 1367.

7. Chernosky ME, Dukes CD. Green nails. Importance of pseudomonas aeruginosa in onychia. Arch Dermatol. 1963; 88: 548-53.

8. Stone OJ, Mullins JF. The role of pseudomonas aeruginosa in nail disease. $J$ Invest Dermatol. 1963; 41: 25-6.

9. Bauer MF, Cohen H. The role of Pseudomonas aeruginosa in infections about the nails. AMA Arch Derm. 1957; 75(3): 394-6.

10. Mermel LA, McKay M, Dempsey J, Parenteau S. Pseudomonas surgical-site infections linked to a healthcare worker with onychomycosis. Infect Control Hosp Epidemiol. 2003; 24(10): 749-52.

11. McNeil SA, Nordstrom-Lerner L, Malani PN, Zervos M, Kauffman CA. Outbreak of sternal surgical site infections due to Pseudomonas aeruginosa traced to a scrub nurse with onychomycosis. Clin Infect Dis. 2001; 33(3): 317-23.

12. Foca M, Jakob K, Whittier S, Della Latta P, Factor S, Rubenstein D, Saiman L. Endemic Pseudomonas aeruginosa infection in a neonatal intensive care unit. N Engl J Med. 2000; 343(10): 695-700.

13. Vergilis I, Goldberg LH, Landau J, Maltz A. Transmission of Pseudomonas aeruginosa from nail to wound infection. Dermatol Surg. 2011; 37(1): 105-6.

14. Corsello G, Vecchio D. Green nail syndrome. Pediatr Int. 2014; 56(5): 801.

15. Chiriac A, Brzezinski P, Foia L, Marincu I. Chloronychia: green nail syndrome caused by
Pseudomonas aeruginosa in elderly persons. Clin Interv Aging. 2015; 10: 265-7.

16. McGinley KJ, Larson EL, Leyden JJ. Composition and density of microflora in the subungual space of the hand. J Clin Microbiol. 1988; 26(5): 950-3.

17. Yang YS, Ahn JJ, Shin MK, Lee MH. Fusarium solani onychomycosis of the thumbnail coinfected with Pseudomonas aeruginosa: report of two cases. Mycoses 2011; 54(2): 16871.

18. Aspiroz C, Toyas C, Robres P, Gilaberte Y. Interacción de Pseudomonas aeruginosa y hongos dermatofitos: repercusión en el curso clínico y en el diagnóstico microbiológico de la tinea pedis. Actas Dermosifiliogr. 2016; 107(1): 78-81.

19. Foster KW, Thomas L, Warner J, Desmond R, Elewski BE. A bipartite interaction between Pseudomonas aeruginosa and fungi in onychomycosis. Arch Dermatol. 2005; 141(11): 1467-8.

20. Larangueira de Almeida Jr H, Pereira Duquia R, Suita de Castro LA, Moreira Rocha N. Scanning electron microscopy of the green nail. Int $\mathbf{J}$ Dermatol. 2010; 49(8): 962-3.

21. Lee H, Mun JH, Cho S, Park H. Clinical analysis of Pseudomonas aeruginosa-positive and negative green nail syndrome cases: A single center retrospective analysis. J Dermatol. 2021; 48(7): 1073-6.

22. Maes M, Richert B, de la Brassinne M. Green nail syndrome or chloronychia. Rev Med Liege. 2002; 57(4): 233-5.

23. Geizhals S, Lipner SR. Retrospective Case Series on Risk Factors, Diagnosis and Treatment of Pseudomonas aeruginosa Nail Infections. Am J Clin Dermatol. 2020; 21(2): 297-302.

24. Ohn J, Yu DA, Park H, Cho S, Mun JH. Green nail syndrome: Analysis of the association with 
Síndrome de la uña verde - Hasbún C. et al.

onychomycosis. J Am Acad Dermatol. 2020; 83(3): 940-2.

25. Ghosh SK, Bandyopadhyay D. The color of skin: green diseases of the skin, nails, and mucosa. Clin Dermatol. 2019; 37(5): 516-9.

26. Gorantla JN, Kumar SN, Nisha GV, Sumandu AS, Dileep C, Sudaresan A et al. Purification and characterization of antifungal phenazines from a fluorescent Pseudomonas strain FPO4 against medically important fungi. J Mycol Med. 2014; 24(3): 185-92.

27. Kerr JR, Taylor GW, Rutman A, Høiby N, Cole PJ, Wilson R. Pseudomonas aeruginosa pyocyanin and 1-hydroxyphenazine inhibit fungal growth. J Clin Pathol. 1999; 52(5): 3857.

28. Treat J, James WD, Nachamkin I, Seykora JT. Growth inhibition of Thrichophyton species by Pseudomonas aeruginosa. Arch Dermatol. 2007; 143(1): 61-4.

29. Bae Y, Lee GM, Sim JH, Lee S, Lee SY, Park YL. Green nail syndrome treated with the application of tobramycin eye drop. Ann Dermatol. 2014; 26(4): 514-6.

30. Cho SB, Kim HS, Oh SH. Green nail syndrome associated with military footwear. Clin Exp Dermatol. 2008; 33(6): 791-3.

31. Rigopoulos D, Rallis E, Gregoriou S, Larios G, Belyayeva Y, Gkouvi K et al. Treatment of pseudomonas nail infections with octenidine dihydrochloride solution. Dermatology. 2009; 218(1): 67-8.

32. Agger WA, Mardan A. Pseudomonas aeruginosa infections of intact skin. Clin Infect Dis. 1995 ; 20(2): 302-8.

33. Romaszkiewicz A, Slawinska M, Sobjanek M, Nowicki RJ. Nail dermoscopy (onychoscopy) is useful in diagnosis and treatment follow-up of the nail mixed infection caused by Pseudomonas aeruginosa and Candida albicans. Postepy Dermatol Alergol. 2018; 35(3): 327-9.
34. Matsuura H, Senoo A, Saito M, Hamanaka Y. Green nail syndrome. QJM. 2017; 110(9): 609.

35. Hengge UR, Bardeli V. Images in clinical medicine. Green nails. N Engl J Med. 2009; 360(11): 1125.

36. Müller S, Ebnöther M, Itin P. Green Nail Syndrome (Pseudomonas aeruginosa Nail Infection): Two Cases Successfully Treated with Topical Nadifloxacin, an Acne Medication. Case Rep Dermatol. 2014; 6(2): 180-4.

37. Rallis E, Paparizos V, Flemetakis A, Katsambas A. Pseudomonas fingernail infection successfully treated with topical nadifloxacin in HIV-positive patients: report of two cases. AIDS. 2010; 24(7): 1087-8.

38. Schwartz RA, Kapila R. The Goldman-Fox syndrome: Treating and preventing green pseudomonas nails in the era of COVID-19. Dermatol Ther. 2020: e14624. 